\title{
SPIRAL ARM MORPHOLOGY IN CLUSTER ENVIRONMENT
}

\author{
IsAaC Yeoun-Gyu Choi ${ }^{1}$, Hong Bae $\mathrm{AnN}^{2}$ \\ ${ }^{1}$ Korea Science Academy of KAIST, Pusan 614-822, Korea \\ E-mail : caasi2@naver.com \\ ${ }^{2}$ Department of Earth Science Education, Pusan National University, Pusan 609-735, Korea \\ E-mail : hbann@pusan.ac.kr \\ (Received July 13, 2011; Revised August 06, 2011; Accepted August 10, 2011)
}

\begin{abstract}
We examine the dependence of the morphology of spiral galaxies on the environment using the KIAS Value Added Galaxy Catalog (VAGC) which is derived from the Sloan Digital Sky Survey (SDSS) DR7. Our goal is to understand whether the local environment or global conditions dominate in determining the morphology of spiral galaxies. For the analysis, we conduct a morphological classification of galaxies in 20 X-ray selected Abell clusters up to $\mathrm{z} \sim 0.06$, using SDSS color images and the X-ray data from the Northern ROSAT All-Sky (NORAS) catalog. We analyze the distribution of arm classes along the clustercentric radius as well as that of Hubble types. To segregate the effect of local environment from the global environment, we compare the morphological distribution of galaxies in two X-lay luminosity groups, the low- $L_{x}$ clusters $\left(L_{x}<0.15 \times 10^{44} \mathrm{erg} / \mathrm{s}\right)$ and high- $L_{x}$ clusters $\left(L_{x}>1.8 \times 10^{44} \mathrm{erg} / \mathrm{s}\right)$. We find that the morphology-clustercentric relation prevails in the cluster environment although there is a brake near the cluster virial radius. The grand design arms comprise about $40 \%$ of the cluster spiral galaxies with a weak morphology-clustercentric radius relation for the arm classes, in the sense that flocculent galaxies tend to increase outward, regardless of the X-ray luminosity. From the cumulative radial distribution of cluster galaxies, we found that the low- $L_{x}$ clusters are fully virialized while the high- $L_{x}$ clusters are not.
\end{abstract}

Key words : galaxies: clusters: general — galaxies: evolution — galaxies: formation — galaxies: morphology

\section{INTRODUCTION}

Spiral arms are the most pronounced feature of galaxies. Two morphological features, openness and patchiness, were adopted as key parameters that divided spiral galaxies into subtypes $\mathrm{Sa}, \mathrm{Sb}$, and $\mathrm{Sc}$ by Hubble (1936). Hubble's morphology classification system, especially the classification of spiral galaxies, was refined by de Vaucouleurs (1959) and Sandage (1961), to make the transition from late type spirals to irregulars smooth by introducing Sd and Sm types, and dividing arm morphologies into ring (r), intermediate (rs), and spiral (s) varieties.

Elmegreen \& Elmegreen (1982) followed a different approach based on the regularity of spiral arm structures and constructed a 12-division classification of spiral arms under two broad categories, flocculent arms and grand design arms. They assigned arm classes (AC) 1-4 to flocculent and 5-12 to grand design arms in their analysis of frequency distribution of arms in field, binary and group galaxies. They found that flocculent arms are most frequently observed in isolated galaxies and galaxies without bars. The connections between grand design arms and the presence of bars were also

Corresponding Author: H. B. Ann observed in cluster galaxies (Elmegreen, Elmegreen, \& Dressler 1982). The grand design galaxies are also likely to found in dense environment and they are bigger than flocculent spirals by a factor of 1.5 (Elmegreen \& Elmegreen 1987). $\mathrm{AC}=10$ and 11 are omitted in the AC systems of Elmegreen \& Elmegreen (1987) because their separation is not solely due to the difference in spiral morphology but due to the presence of companions or bars.

Grand design galaxies are thought to be formed by density waves (Lin \& Shu 1964), while flocculent galaxies are caused by stochastic self-propagating star formation (Mueller \& Arnett 1976; Gerola \& Seiden 1978; Seiden \& Gerola 1982). However, the tides driven by bars or interactions with companion galaxies (Toomre 1972; Tully 1974; Oh et al. 2008) were also suggested as causing grand design spirals. Turbulent motions driven by sheared gravitational instability (Elmegreen \& Elmegreen 2003) or self-regulated star formation (Cartin \& Khanna 2002) are alternative explanation for the formation of the flocculent spirals. Recently, Dobbs et al. (2010) report that a tide induced density wave model fits the observations of M51 quite well.

Elmegreen (1990) found that grand design galaxies (AC 12) are likely to have falling rotation curves, while 


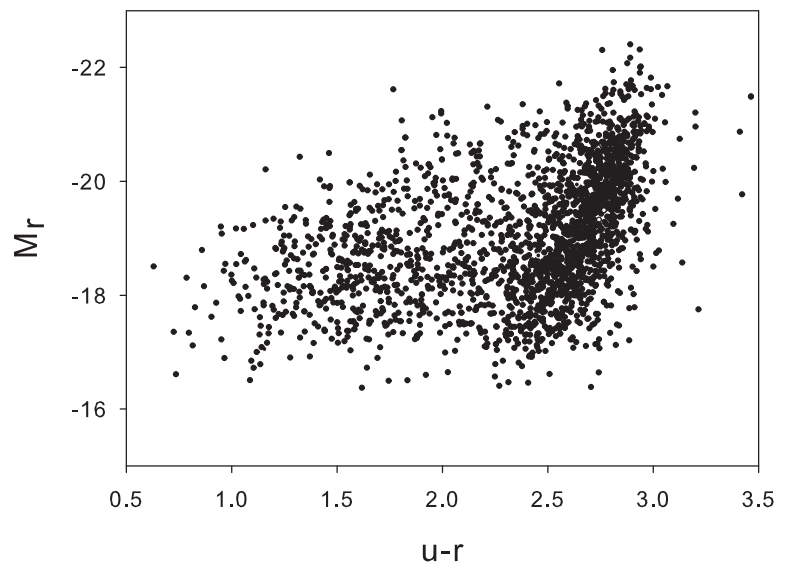

Fig. 1. $-M_{r}$ versus $u-r$ color diagram of cluster galaxies.

flocculent (AC 1-4) and multiple arm (AC 5-9) galaxies show nearly flat or rising rotation curves. It is worthwhile to note that they divided the previous category of grand design (AC 5-12) into multiple arm (AC 5-9) and grand design (AC 12) to segregate symmetric arms in galaxies with falling rotation curves from those in galaxies with flat or rising rotation curves.

Dressler (1980) was the first to study the effect of the environment on galaxy morphology. He analyzed the morphology of galaxies in 55 rich clusters of galaxies and found that elliptical and S0 galaxies are likely to be found in high density regions while spiral galaxies are preponderant in low density regions. The morphology-local density relations were observed in poor clusters (Postman \& Geller 1984) as well as in the X-ray selected groups (Tran et al. 2001; Helsdon \& Ponman 2003). However, Whitmore (1995) argued that the morphology-local density relation is very weak in groups. In addition, this relation was observed not only in cluster galaxies, but in field galaxies as well (Goto et al. 2003; Park et al. 2007).

Local background density is usually determined by counting the number of galaxies around a target galaxy (e.g., Muldrew et al. 2011). However, various other density parameters have also been used for investigating the relation between galaxy properties and their environment. For example, Gray et al. (2004) used the dark matter density while Wolf et al. (2009) used stellar mass density as a parameter.

Furthermore, the morphology of galaxies is known to depend not only on local background density but on the distance from the center of clusters. The morphologyclustercentric radius relation also seems to operate in large systems such as cluster of galaxies as well as in small systems, i.e., satellite systems of galaxies (Ann, Park, \& Choi 2008). Thus, the underlying mechanisms that govern the morphology-local density re- lation and the morphology-clustercentric radius relation seem to be different. Between these two relationships, Whitmore \& Gilmore (1991) argued that the morphology-clustercentric radius relation is more fundamental than the morphology-local density relation because morphologies of galaxies are more tightly correlated with the clustercentric radius than the local background density. Thomas \& Katgert (2006) also analyzed 850 galaxies in 23 clusters and investigated both the morphology-local density relation and the morphology-clustercentric radius. The unique aspect of their research is that they divided the elliptical galaxies into bright ellipticals and normal ellipticals. They concluded that bright ellipticals and late spirals seem to follow the morphology-clustercentric radius relation while the morphology-local density relation is seen among normal ellipticals, S0 galaxies and early type spirals.

Aside from clustercentric radius and local density, astronomers made efforts to relate the morphology of galaxies with other physical parameters such as mass, luminosity, star formation activities and even neighbors. Postman et al. (2005) measured several $\mathrm{z} \sim 1$ clusters and concluded that the bolometric X-ray luminosity of the intracluster medium is related with the morphology-local density relation. Park \& Hwang (2009) investigated the dependence between morphology of the galaxies with the nearest neighbor galaxy and concluded that the distance and morphology of the nearest neighbor has a significant influence on the overall morphology of galaxies. Balogh et al. (2002) also investigated the influence of X-ray luminosity of a cluster to fractional bulge luminosity $(\mathrm{B} / \mathrm{T})$, by dividing the clusters into low and high luminosity groups, and concluded that the destruction of disks through ram-pressure stripping or harassment is not solely responsible for the morphology-local density relation and that bulge formation is less efficient in low-mass clusters. However this study did not see the dependence of Hubble morphology on the local density. van den Bergh (2002) showed that the frequency of spiral arm varieties, (r), (rs), and (s), does not depend on the environment but it does depend on the internal properties of a galaxy.

Adopting the classification system of Elmegreen \& Elmegreen (1982), we aim to understand the key factors that affect the spiral morphology of galaxies in cluster environments, especially the arm classes. We also intend to analyze the dependence of the morphologyclustercentric radius relation on the X-ray luminosity of the clusters.

In Section 2, we introduce the observational data we use, and the procedure of extraction of the pure samples and list the physical factors that we will consider. We present our results in Section 3 and discussions follow in Section 4. We present our conclusions in Section 5 . 

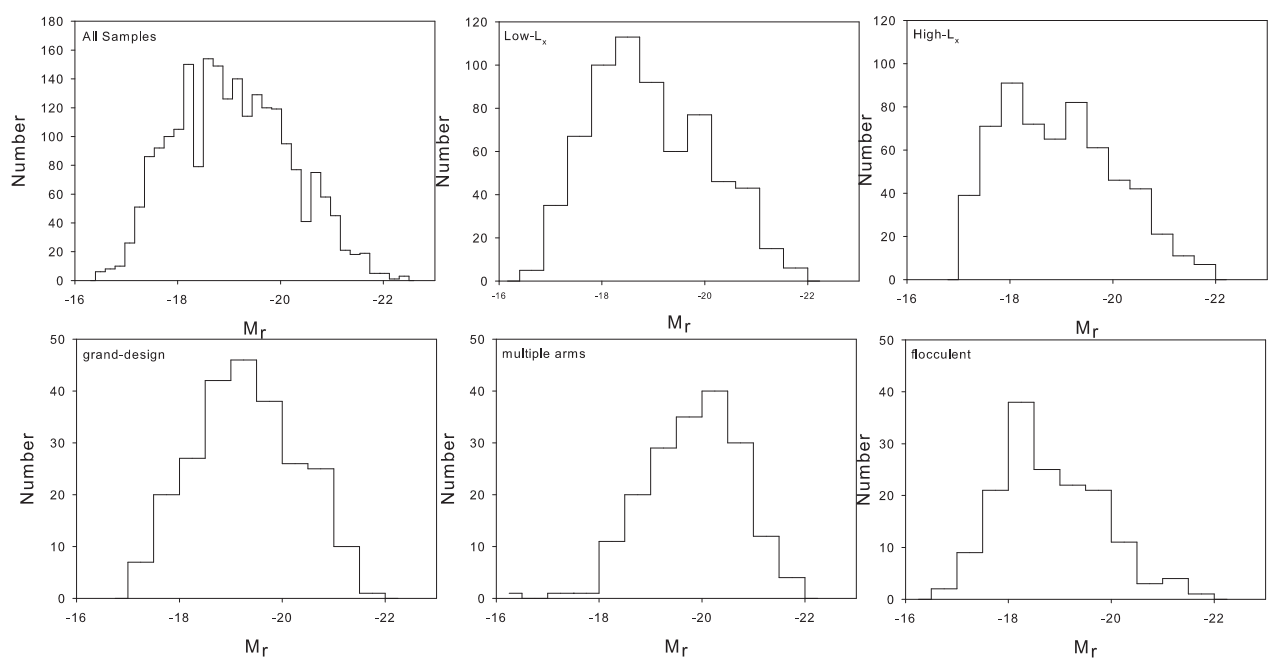

Fig. 2.- The number distributions of cluster galaxies as function of $M_{r}$. The full sample, galaxies in the low- $L_{x}$ clusters and those in the high- $L_{x}$ clusters are plotted in the upper panels, from left to right. In the lower panels, the number distributions of arm classes are given from left to right, in the order of grand design, multiple arms, and flocculent.

\section{OBSERVATIONAL DATA}

\subsection{KIAS VAGC and Sloan Digital Sky Survey}

We used the photometric data and redshifts of KIAS VAGC (Choi, Han, \& Kim 2010) which is a value-added catalog of galaxies based on the New York University Value-Added Galaxy Catalog (NYU VAGC) Data Release 7. KIAS VAGC supplements red shifts of 10,497 galaxies to the NYU VAGC LSS sample, which again come from Sloan Digital Sky Survey (SDSS) Data Release 7(DR7; Abazajian et al. (2009)). The survey contains five-band(ugriz) photometric data for 230 million objects over $8400 \mathrm{deg}$ and optical spectroscopic data more than one million objects of galaxies, quasars, and stars over $6860 \mathrm{deg}$ (Gunn et al. 1998; Uomoto et al. 1999; Castander et al. 2001; Blanton et al. 2003; Fukugita et al. 1996; Pier et al. 2003; Tucker et al. 2006). More precise descriptions of SDSS data are given by York et al. (2000) and Stoughton et al. (2002).

\subsection{Cluster Sample and Galaxy Membership}

We selected our samples among the Abell catalog of galaxy clusters (Abell, Corwin, \& Olowin 1989) (Abell et al. 1989) and the Northern ROSAT All-Sky (NORAS) galaxy cluster survey (Bohringer et al. 2000) The clusters that were in both catalogs (surveys) were then examined if they were in the SDSS survey region, and had redshifts below $\mathrm{z} \sim 0.06$, the maximum redshift we considered for the least recognizable resolution of SDSS photometric data. We found 21 galaxy clusters that satisfy these conditions. Abell 1825 was then excluded because it did not have adequate number of member galaxies, leaving us with 20 clusters. We present the coordinates, red shifts, X-ray luminosity and other important properties of the 20 Abell clusters in Table 1.
To find out the galaxies that are included in the clusters, we used the shifting gaper method (Fadda et al. 1996) which is better than the fixed gap method at removing interlopers. We looked for galaxies around a target cluster using a bin size of $0.4 h^{-1} \mathrm{Mpc}$ and velocity width of $1000 \mathrm{~km} / \mathrm{s}$ to select the member galaxies. The maximum radius for the galaxy search was $2 h^{-1} \mathrm{Mpc}$ which is larger than the virial radii of the typical clusters in our sample. We used the coordinates of the cluster center given in Table 1, which are those obtained from the SDSS.

We then calculated the virial radius, which usually denotes the radius within which the cluster obeys the virial theorem. However, we use a practical definition of the virial radius as the radius within which the mean density drops to 200 times the critical density of the universe. The formula for this radius is Carlberg, Yee, \& Ellingson (1997),

$$
R_{v i r}=\frac{3^{1 / 2} \sigma_{d}}{10 H(z)}
$$

where $\sigma_{d}$ is the velocity dispersion of the cluster and the $H(z)$ is the Hubble parameter at redshift $\mathrm{z}$, which can be written as $H^{2}(z)=H_{0}{ }^{2}\left(\Omega_{m}(1+z)^{3}+\Omega_{k}(1+\right.$ $\left.z)^{2}+\Omega_{\Lambda}\right)$ (Peebles 1993), here $H_{0}$ is the Hubble constant and $\Omega_{m}, \Omega_{k}$, and $\Omega_{\Lambda}$ are the dimensionless density parameters. We used $H_{0}=100 \mathrm{~km} / \mathrm{s}, \Omega_{m}=0.3$, $\Omega_{k}=0$, and $\Omega_{\Lambda}=0.7$. Though we searched for member galaxies within $2 h^{-1} \mathrm{Mpc}$ from the cluster center, we derived the velocity dispersion of the cluster from galaxies inside the virial radius. 
Table 1.

Properties of the Clusters

\begin{tabular}{cccccccc}
\hline \hline Name & RA & Dec & $\mathrm{z}$ & $\mathrm{N}^{a}$ & $R_{\text {vir }}{ }^{b}$ & $\sigma^{c}$ & $L_{x}(0.1-2.4 \mathrm{keV})^{d}$ \\
\hline $\mathrm{A} 0152$ & 17.460833 & 13.982778 & 0.0581 & 66 & 1.02 & 604 & 0.14 \\
$\mathrm{~A} 0160$ & 18.214167 & 15.515000 & 0.0447 & 37 & 2.35 & 1382 & 0.37 \\
$\mathrm{~A} 0168$ & 18.790833 & 0.247500 & 0.0450 & 111 & 1.10 & 644 & 0.92 \\
$\mathrm{~A} 0671$ & 127.122083 & 30.416944 & 0.0502 & 100 & 1.02 & 602 & 0.78 \\
$\mathrm{~A} 0757$ & 138.197083 & 47.710556 & 0.0517 & 55 & 0.63 & 370 & 0.5 \\
$\mathrm{~A} 0779$ & 139.961667 & 33.771389 & 0.0225 & 44 & 2.86 & 1664 & 0.06 \\
$\mathrm{~A} 1100$ & 162.22625 & 22.235556 & 0.0463 & 72 & 0.72 & 422 & 0.12 \\
$\mathrm{~A} 1185$ & 167.699167 & 28.678333 & 0.0325 & 168 & 1.62 & 949 & 0.00 \\
$\mathrm{~A} 1139$ & 164.517917 & 1.498889 & 0.0398 & 115 & 0.55 & 322 & 0.15 \\
$\mathrm{~A} 1142$ & 165.229167 & 10.547778 & 0.0349 & 58 & 1.06 & 621 & 0.28 \\
$\mathrm{~A} 1177$ & 167.365833 & 21.695278 & 0.0316 & 53 & 0.79 & 463 & 0.17 \\
$\mathrm{~A} 1291$ & 173.01875 & 56.023889 & 0.0527 & 67 & 1.40 & 463 & 0.51 \\
$\mathrm{~A} 1314$ & 173.702917 & 49.040278 & 0.0335 & 70 & 2.71 & 948 & 0.14 \\
$\mathrm{~A} 1367$ & 176.122917 & 19.839167 & 0.022 & 163 & 2.55 & 1485 & 1.51 \\
$\mathrm{~A} 1377$ & 176.74125 & 55.738889 & 0.0514 & 107 & 1.05 & 619 & 0.36 \\
$\mathrm{~A} 1991$ & 223.625833 & 18.630833 & 0.0587 & 96 & 1.03 & 610 & 1.46 \\
$\mathrm{~A} 2052$ & 229.189583 & 7.000278 & 0.0355 & 136 & 2.23 & 1305 & 2.58 \\
$\mathrm{~A} 2063$ & 230.757917 & 8.639444 & 0.0349 & 156 & 2.18 & 1273 & 1.94 \\
$\mathrm{~A} 2197$ & 247.04375 & 40.907222 & 0.0308 & 242 & 1.10 & 640 & 0.13 \\
$\mathrm{~A} 2199$ & 247.160417 & 39.551667 & 0.0302 & 316 & 0.95 & 557 & 3.77 \\
\hline
\end{tabular}

$a$ the number of galaxies within $2 h^{-1} \mathrm{Mpc}, \quad{ }^{b}$ virial radius of cluster in $h^{-1} \mathrm{Mpc}$,

${ }^{c}$ velocity dispersion of the cluster in units of $\mathrm{km} / \mathrm{s}$,

$d$ X-ray luminosity $(0.1-2.4 \mathrm{keV})$ of the cluster from the NORAS survey in units of $10^{44} \mathrm{erg} / \mathrm{s}$.

\subsection{Morphology and Physical Parameters}

Since we aim to understand the relationship between the morphological features of cluster galaxies and the local and global environment parameters of the clusters, it is crucial to determine the morphological types accurately. We considered the X-ray luminosity and the clustercentric radius normalized by the virial radius of the cluster as the global and local environment parameters, respectively. The physical parameters characterizing the morphology of spiral galaxies were taken to be the bar type, Hubble type and the arm class.

Based on the SDSS color images, the spiral galaxies were visually examined to determine the bar type, Hubble type and arm classes. The arm classes were adopted from (Elmegreen \& Elmegreen 1987) and simplified, following (Elmegreen 1990), as flocculent (AC 1-4), multiple armed (AC 5-9) and grand design (AC 12). For bar type, we divided spiral galaxies into three types SA, SAB and SB based on bar strength. For Hubble types, we divided galaxies into ellipticals, lenticulars, spirals and irregulars with further subdivisions into early ( $\mathrm{Sa}, \mathrm{Sab}, \mathrm{Sb})$, intermediate (Sbc, Sc, $\mathrm{Scd})$, and late (Sd, Sdm, Sm) types. Galaxies that are edge-on were treated with more care. Hubble types of edge-on galaxies were determined by their bulge sizes and color gradients. While analyzing bar types, we excluded edge-on galaxies because their bars are not distinguishable. These morphological parameters were checked again with 2 -week intervals to reduce personal error. Although we have classified bar types along with the Hubble type and arm classes, we do not use the bar types in the present study. In our forthcoming papers, we will report on the bar related properties of cluster galaxies. We present the sample images to show the spiral morphology (Bar, T, AC) for bright galaxies $(r<15)$ and faint galaxies $(r>16.5)$ in the appendix.

$\mathrm{X}$-ray luminosity of the galaxy clusters were adopted from the NORAS catalog. For quantitative analysis of the clusters, we divide the clusters into three groups as low- $L_{x}$ group $\left(L_{x}<0.15 \times 10^{44} \mathrm{erg} \mathrm{s}^{-1}\right)$, intermediate$L_{x}$ group and high- $L_{x}$ group $\left(L_{x}>1.8 \times 10^{44} \mathrm{erg} \mathrm{s}^{-1}\right)$. However, we used only low- $L_{x}$ group and high- $L_{x}$ group in the analysis of the X-ray luminosity dependence of galaxy morphology. We adopted this division because of the lack of clusters comparable to the high X-ray luminous clusters used by Balogh et al. (2002). In the above division, 6 clusters belong to the low- $L_{x}$ group and 3 clusters belong to high- $L_{x}$ group. The numbers of galaxies in the low- $L_{x}$ and high- $L_{x}$ groups are 662 

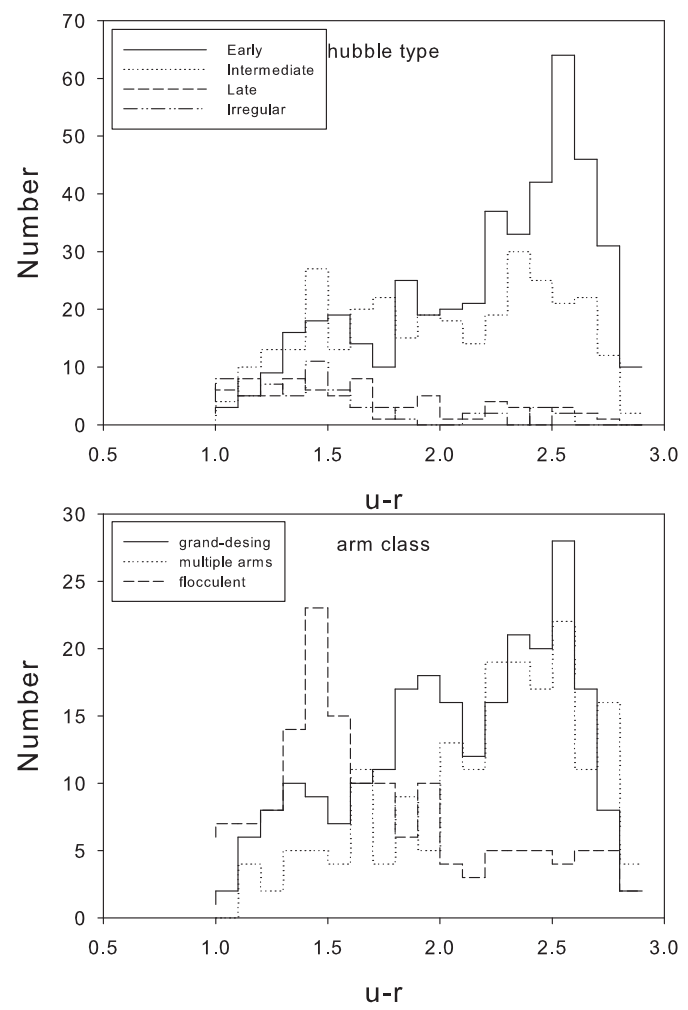

Fig. 3. - The number distributions of spiral galaxies in clusters as a function of $\mathrm{u}-\mathrm{r}$ color. The upper panel shows the distributions of the Hubble types while the lower panel displays the arm classes.

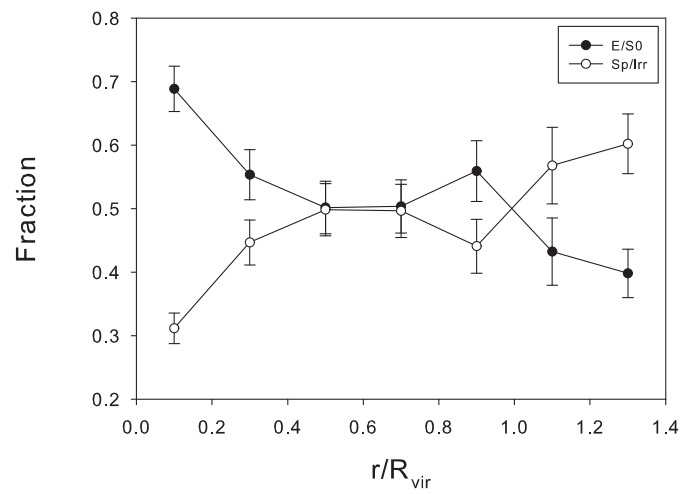

Fig. 4. - The fraction of elliptical and S0 galaxies (open circle) and the spirals and irregular galaxies (solid circle) as a function of the clustercentric radius normalized to the cluster virial radius $\left(R_{v i r}\right)$. The bin is $0.2 R_{v i r}$. The error bar indicates the Poisson errors.
Table 2.

Number of spiral galaxies in bar types, Hubble types, and arm classes.

\begin{tabular}{cccc}
\hline \hline Type $^{a}$ & 1 & 2 & 3 \\
\hline Bar & 246 & 218 & 226 \\
T & 453 & 363 & 85 \\
AC & 242 & 184 & 157 \\
\hline
\end{tabular}

${ }^{a}$ 1, 2, 3 each represents non barred(A), weakly barred(AB), and barred(B) for bars, and early, intermediate, late types for Hubble types, and grand designs, multiple arms, flocculent for arm classes.

\section{RESULTS}

\subsection{Photometric Properties of Galaxies}

Fig. 1 demonstrates the relationship between $u-r$ color and absolute magnitude $\left(M_{r}\right)$ of the galaxies. This graph shows both the red sequence and the blue cloud which are seen in field galaxies showing that the photometric properties of the cluster galaxies are similar to those of the field galaxies (de Vaucouleurs 1961). Fig. 2 shows the distribution of $M_{r}$ of galaxies, grouped by X-ray luminosity and arm classes. The full sample presented in the upper left panel of Fig. 2 shows a peak at $M_{r} \approx-19.0$.However, if we divide the sample based on the arm classes, the peaks are seen to differ. In the order of multiple arms, grand design, flocculent, the luminosity of the peak gets dimmer. However the $M_{r}$ distribution is not affected by the X-ray luminosity of the clusters. This implies that the luminosity function of cluster galaxies does not depend much on the cluster luminosity, at least in the present sample, which lacks high X-ray luminosity clusters.

Table 2 shows the overall number of the morphological parameters of spiral galaxies we classified. While bars are distributed evenly, Hubble types tend to be early and intermediate. Arm classes also have less flocculent galaxies. We can also see that the number of galaxies whose Hubble types were identified is larger than the number of galaxies whose bar type was identified. This is because we excluded edge-on spirals in the process of bar classification.

Fig. 3 shows the relationship between these morphological properties with the $u-r$ color of the galaxies. The late type spirals and irregulars show a peak at a smaller $u-r$ color compared to the early and intermediate types. A similar feature is seen in the flocculent arm class as well. This shows that late, flocculent spirals along with irregulars tend to be bluer than other types of galaxies.

and 608 , respectively. 

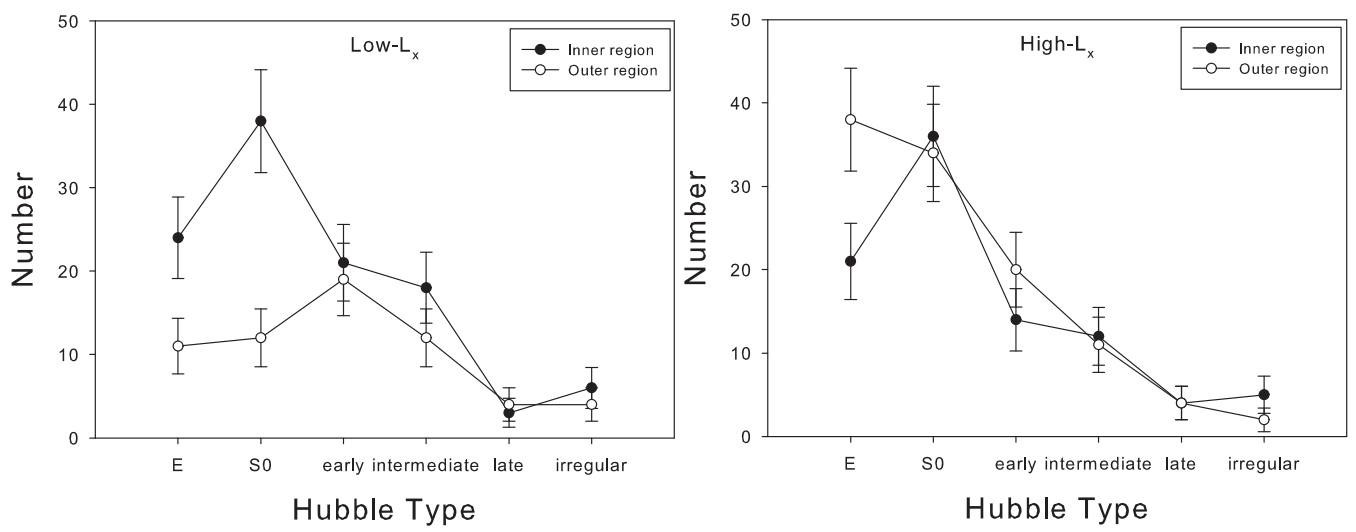

Fig. 5. - The number distribution of the Hubble types of the low- $L_{x}\left(<0.15 \times 10^{44} \mathrm{erg} / \mathrm{s}\right)$ samples (left panel) and high- $L_{x}$ $\left(>1.8 \times 10^{44} \mathrm{erg} / \mathrm{s}\right)$ samples (right panel) for the inner region $\left(0.2<r / R_{v i r}<0.4\right.$, thick line with solid circles) and outer region $\left(0.8<r / R_{v i r}<1.0\right.$, thin line with open circles). The error bar represents Poisson errors.

\subsection{Morphology-Radius Relation of Cluster Galaxies}

Following arguments that the morphology-radius relation is more fundamental than the morphologydensity relation of galaxies (Whitmore \& Gilmore 1991), we adopt the projected clustercentric radius divided by the cluster virial radius $\left(r / R_{v i r}\right)$ as an independent parameter. The reason why we used normalized radius rather than physical radius in units of Mpc is that the physical size of a cluster correlates with its mass. Fig. 4 shows the fraction of early type galaxies (E/S0) and late type galaxies (Sp/Irr) as a function of $r / R_{v i r}$. It is seen that the early type fraction decreases steadily from the central regions to the outer regions while the late type galaxies increases outward. This trend agrees with the earlier findings of Dressler (1980) and Whitmore \& Gilmore (1991).

However, since most earlier studies use the physical radius in units of $\mathrm{Mpc}$, a direct comparison of the distribution of galaxy morphology is difficult. We compared our results with Park \& Hwang (2009) who used the same independent parameter $\left(r / R_{v i r}\right)$ as ours for the analysis of 200 Abell Clusters of galaxies which have more than 10 member galaxies. Since the basic photometric and redshift data of Park \& Hwang (2009) are virtually the same as our study, the excellent match of their early type fractions of 0.5 and 0.4 for galaxies of $-17.0 \geq M_{r}>-19.0$ at $r / R_{v i r}=0.5$ and $r / R_{\text {vir }}=1.3$, respectively with those of the present results shown in Fig. 4 is not surprising. We determined morphological types visually, while Park \& Hwang (2009) employed Park \& Choi (2005)'s automated morphology classifier which shows an accuracy of $85 \sim 90 \%$ for most of cases. We also compared the morphological types in KIAS VAGC with our visual classification and found that the morphological types match for $\geq 85 \%$ of galaxies.

\subsection{X-Ray Luminosity and Morphology-Radius Relation}

In order to understand which environment (local or global) is more responsible for the morphology of cluster galaxies, we performed an analysis similar to that in Balogh et al. (2002) who analyzed the frequency distribution of cluster galaxies as a function of $B / T$ by considering X-ray luminosity as characterizing the global environment and the background density as the variable characterizing the local environment. Fig. 5 shows the number distributions of galaxies in the two representative regions as a function of Hubble types for the two groups of clusters selected by their X-ray luminosity. We chose the inner and outer regions at $r=(0.2 \sim 0.4) R_{v i r}$ and $r=(0.8 \sim 1.0) R_{v i r}$, respectively, by considering the radial distribution of galaxies as a function of $r / R_{v i r}$.

The frequency distributions of Hubble types in the inner regions of the clusters are similar to each other, regardless of the cluster X-ray luminosities. Moreover, if we consider only the late type galaxies, there is no significant difference in the frequency distributions between the inner regions and the outer regions, regardless of the cluster X-ray luminosities. This means that neither the local environment nor the global environment affects the late type populations. The combined effect of the local and global environment inside the cluster virial radius results in an increase in the number of galaxies from the late type spirals including irregular galaxies to the early type spirals. This trend appears to extend to the lenticular galaxies except for the outer regions of the low- $L_{x}$ clusters.

However, the frequency distribution of early type galaxies seems to be significantly affected by both the local and the global environment in a manner that is difficult to disentangle. In the low- $L_{x}$ clusters, the number of elliptical galaxies in the inner regions is about two times larger than that in the outer regions, 
Table 3.

Physical parameters of cluster galaxies with their morphological types*

\begin{tabular}{|c|c|c|c|c|c|c|c|c|c|c|c|c|}
\hline Name $^{a}$ & $\mathrm{RA}$ & Dec & $\mathrm{z}$ & $\mathrm{d}\left(h^{-1} \mathrm{Mpc}\right)$ & $M_{r}$ & $u-r$ & $r\left(h^{-1} \mathrm{Mpc}\right)$ & $r / R_{v i r}$ & $\operatorname{Bar}^{b}$ & $\mathrm{~T}^{c}$ & $\mathrm{AC}^{d}$ & flag $^{e}$ \\
\hline 55175 & 164.125 & 0.987823 & 0.039432 & 117 & -19.491 & 2.159 & 1.331 & 2.42 & 3 & 1 & 2 & 0 \\
\hline 55178 & 164.195389 & 0.922453 & 0.040448 & 120 & -19.029 & 1.749 & 1.364 & 2.48 & 1 & 2 & 0 & 0 \\
\hline 55186 & 164.631683 & 0.904621 & 0.040099 & 119 & -19.165 & 2.137 & 1.250 & 2.27 & 1 & 0 & 0 & 0 \\
\hline 55187 & 164.644363 & 1.037104 & 0.040164 & 119 & -18.223 & 1.594 & 0.989 & 1.79 & 1 & 4 & 0 & 0 \\
\hline 55195 & 165.011719 & 1.038455 & 0.039634 & 117 & -17.889 & 1.438 & 1.394 & 2.53 & 1 & 4 & 0 & 0 \\
\hline 79351 & 164.071091 & 0.677375 & 0.040498 & 120 & -18.466 & 1.106 & 1.932 & 3.51 & 1 & 1 & 0 & . \\
\hline 79357 & 164.34465 & 0.661611 & 0.039100 & 116 & -19.299 & 2.408 & 1.766 & 3.21 & 0 & 0 & 0 & 0 \\
\hline 79361 & 164.304901 & 0.638563 & 0.039282 & 116 & -19.066 & 2.103 & 1.831 & 3.32 & 1 & 1 & 0 & . \\
\hline 79376 & 164.720337 & 0.679703 & 0.039463 & 117 & -19.929 & 1.961 & 1.743 & 3.16 & 3 & 1 & 1 & 1 \\
\hline 79377 & 164.837997 & 0.807692 & 0.039800 & 118 & -19.967 & 2.822 & 1.573 & 2.86 & 0 & 0 & 0 & 0 \\
\hline
\end{tabular}

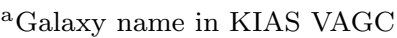

${ }^{\mathrm{b}}$ Bar types coded as 1: SA, 2: SAB, 3: SB

${ }^{\mathrm{c}}$ Hubble types coded as 0: E/S0, 1: early type ( $\left.\mathrm{Sa}-\mathrm{Sb}\right), 2$ : intermediate type ( $\left.S b c-\mathrm{Scd}\right), 3:$ late type $(S d-\mathrm{Sm})$ ), 4: Irr

d Arm classes coded as 1: grand design 2: multiple armed, 3: flocculent. We used 0 for unclassifiable one.

edge-on flag coded as 0: normal, 1: Edge-on

*the full data is given at http://earth.es.pusan.ac.kr/hbann/jkas/v44/table3

whereas the opposite is true for the high- $L_{x}$ clusters. Since the preponderance of early type galaxies in the inner regions is expected from the morphology-radius relation as shown in Fig. 4, the observation that the number of early type galaxies (E/S0) in the outer regions of the X-ray luminous clusters is larger than that in the inner regions of the $\mathrm{X}$-ray luminous clusters, is somewhat confusing. The reason for the excess number of early type galaxies in the outer regions of the X-ray luminous clusters is not yet understood, but it seems evident that the global environment does play a role in the formation and evolution of early type populations.

Comparing Fig. 5 with Fig. 6 of Balogh et al. (2002), we do find that there is an agreement between the present study and Balogh et al. (2002), in the sense that there is no significant dependence of the frequency distribution of late type galaxies (spirals and irregulars) on the local as well as global environment. However, the dependence of the early type fractions on the local and global environment is different. In the present study, the low- $L_{x}$ clusters show a large difference in the frequency of lenticular galaxies between the inner regions and the outer regions, while Balogh et al. (2002) show a similar distribution in the low- $L_{x}$ clusters.

The causes for the differences between our results and Balogh et al. (2002) are partly due to the different independent variables. We used morphological types while Balogh et al. (2002) used $B / T$. We suspect that their use of the local background density as the independent variable does not capture the dependence on the local environment compared to the clustercentric radius. As argued by Whitmore \& Gilmore (1991) morphology-clustercentric radius seems to be more fundamental than the morphology-density relation since the latter relation holds for smaller scales such as the galactic satellite systems which are located in a wide range of the local background density (Ann, Park, \& Choi 2008).

\subsection{Environment Dependence of Spiral Arm Classes}

In order to examine the dependence of arm classes on the global environment, we analyzed the cumulative radial distributions of galaxies for the two $\mathrm{X}$-ray luminosity groups. As shown in Fig. 6, the three arm classes are quite similar in the radial distributions in the low-Lx clusters. However, there is considerable difference between the distribution of grand design and multiple armed galaxies and flocculent galaxies in the high- $L_{x}$ clusters. There is also significant difference in the shape of the radial distributions between the low$L_{x}$ clusters and the high- $L_{x}$ clusters. The Low- $L_{x}$ clusters show nearly a single slope within the virial radius and flat thereafter due to disappearance of galaxies at $r>R_{v i r}$. On the other hand, the high- $L_{x}$ clusters show variable slopes, indicating non-uniform distribution of galaxies. Moreover, the slopes increase steadily until $r \sim 1.5 R_{v i r}$. This means that non-negligible fraction of galaxies are present beyond the virial radius in the high- $L_{x}$ clusters. Thus, we can suggest that the low- $L_{x}$ clusters are already virialized and most of the member galaxies are located inside the virial radius while the 


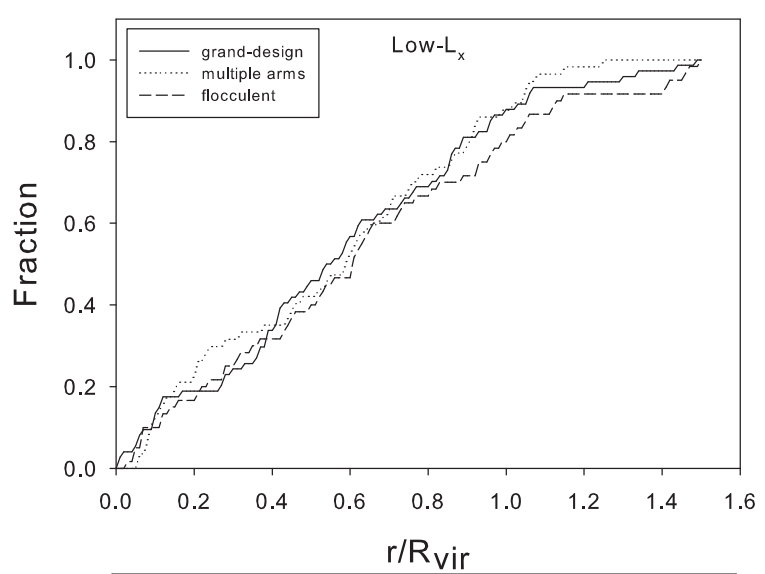

ever, as indicated by the high fraction of grand design galaxies in the outer regions of the high- $L_{x}$ clusters, where the strong tidal interactions between a galaxy and the whole cluster are expected, we can conclude that the global environment does play some role.

The deficiency of flocculent spirals regardless of the local and global environment (shown in Fig. 7) is in good agreement with earlier findings (Elmegreen, Elmegreen, \& Dressler 1982). This is in sharp contrast to the preponderance of flocculent spirals in the isolated field galaxies (Elmegreen \& Elmegreen 1982).

\section{DISCUSSION}

Spiral morphology is characterized by two parameters. One is intimately related to the basic structural parameter $B / T$ which is highly correlated with the openness of the spiral arms and the other is related to the mode of star formation which is thought to be related to the arm classes. While most earlier studies tried to explain the dependence of the structural parameter $(B / T)$ on the environment, revealed by the morphology-density relation or morphology-radius relation, we attempt to explain the dependence of arm classes on the local and global environment of galaxies.

\subsection{The Effects of Global and Local Environ- ment}

The morphology of a galaxy obeys the morphologydensity relation (Dressler 1980) or morphology-radius relation (Whitmore \& Gilmore 1991), both of which represent the dependence of the morphology on the local environment. As shown in Fig. 4, the present sample of nearby Abell clusters $(z<0.06)$ obey the morphology-clustercentric radius relation which dictates the decrease of early type fractions along the clustercentric radius

However, as suggested in Fig. 5, the early type fractions do not seem to decrease monotonously outward in the high- $L_{x}$ clusters while they obey the morphologyradius relation in the low- $L_{x}$ clusters. This means that global environment, represented by X-ray luminosity may play some role for the formation of early type galaxies. The reason for the importance of the global environment is easily understood if we consider that the local background density approaches that of a general field outside the virial radius of any cluster. Since we divide the clusters based on the X-ray luminosity which is thought to be well correlated with the total mass of the cluster, the cluster tidal field near the boundary of the virial radius is stronger in the high luminosity group than in the low luminosity group. Since tidal interactions can transform the late type morphology to the early type ones (Spitzer \& Baade 1951; Merritt 1983), clusters with high X-ray luminosity provide favorable environment for the formation of the early type galaxies even in the outer regions of clusters.

But, for late type galaxies, the Hubble type frac- 
tion does not show any dependence on the local and global environment. The best that can be said is that early type spirals are more frequent than the late type ones in the cluster environment. This conclusion is somewhat different from the flat distribution of $B / T$ in the low- $L_{x}$ clusters (Balogh et al. 2002) although their low- $L_{x}$ clusters include all the clusters with $L_{x}<10^{44}$ $\mathrm{erg} / \mathrm{s}$ while our low- $L_{x}$ clusters have $L_{x}<0.15 \times 10^{44}$ $\mathrm{erg} / \mathrm{s}$. Moreover, in contrast to the almost similar fraction of lenticular galaxies in the inner and outer regions of the high- $L_{x}$ clusters, they showed that the fraction of lenticular galaxies is higher in the high density regions of the clusters with $L_{x}>10^{45} \mathrm{erg} / \mathrm{s}$. Thus, the trend is different in the X-ray luminous clusters.

The frequency distribution of arm types is not seen to be significantly affected by the local environment. However, we observe that the fraction of grand design spirals is much higher than those of other arm classes except in the outer regions of the low- $L_{x}$ clusters where the difference between the fraction of grand design and that of other arm classes is the least (Fig. 7). This implies that the grand design spirals are preferentially formed in regions where galaxy-galaxy and/or galaxycluster tidal interactions are supposed to be high. Since galaxy-galaxy interactions are mostly depend on the local background density while galaxy-cluster interactions depend on the total mass of the cluster, the global environment as well as the local environment seem to affect the arm class distributions.

\subsection{Morphology-Radius Relations for Hubble Type and Arm Class}

The Hubble types obey the morphology-clustercentric radius relation as shown in Fig. 4. However, there is some noise in the relationship such as a bump in the early type fraction or dip in the late type fraction. Since we have already seen that the frequency distribution of Hubble types depends on the global environment, noise can be reduced if we plot it for each X-ray luminosity group.

Fig. 8 shows the morphology-clustercentric radius relation for the low- $L_{x}$ clusters in the left panel and the high- $L_{x}$ clusters in the right panel. As we expected the morphology-radius relation is well revealed for the low- $L_{x}$ clusters inside cluster virial radius. The morphological fractions beyond virial radius approach the field fractions. But the distribution of early type fraction of the high- $L_{x}$ clusters shows a strange behavior for $0.5<r / R_{v i r}<1$. The early type fractions decrease monotonously outward until $r / R_{v i r} \approx 0.5$ and thereafter it increases outward with a peak at $r / R_{v i r} \approx 0.9$. It becomes equal to the mean field fraction at $r / R_{v i r} \approx 1.5$. This means that the size of the $\mathrm{X}$-ray luminous clusters well exceed the cluster virial radius.

The peak at $r / R_{v i r} \approx 0.9$ does not appear to be a real feature because it is greatly reduced when use small binning size $\left(\sim 0.1 h^{-1} \mathrm{Mpc}\right)$ in the shift gaper method. However, there is some possibility that the morphology of infalling galaxies maybe altered by tidal and hydrodynamic interaction resulting in an increase of early type fractions near the virial radius of the cluster. Since most of the mechanisms proposed to remove gas from the disks of spiral galaxies are affected by the total cluster mass or temperature of the hot intracluster medium (see Park \& Hwang (2009) for a detailed discussion of the gas removal mechanisms), the global environment does play a role in the morphology distribution of cluster galaxies, especially in the luminous clusters.

As discussed above, it is evident that the spiral arm classes depend on the local and global environment. However, as shown in Fig. 9, the dependence of arm classes on the local environment is not as strong as that for the Hubble types. More precisely, the radial variation of the fractions of arm classes is very weak even in the low- $L_{x}$ clusters which show strong morphologyclustercentric radius relation for Hubble types. One thing to note is that the fraction of flocculent spirals tends to increase outward in both cluster samples. This implies that the formation of flocculent spirals is suppressed by tidal field, the stronger the tidal field, larger is the suppression of the flocculent spirals. This is supported by the fact that the fraction of flocculent spirals is on average $\sim 50 \%$ lower in the high- $L_{x}$ clusters (Fig. 9 ). We also suspect that the higher temperature of the intracluster gas in the high- $L_{x}$ leads to suppression of the small scale instabilities that would lead to stochastic star formation.

The combined fraction of grand design and multiple arm spirals shown as dotted lines in Fig. 9 is $\sim 0.7$ in the low- $L_{x}$ clusters and $\sim 0.8$ in the high- $L_{x}$ clusters, revealing the effect of the global environment on the arm classes. The present fractions of arm classes agree well with those of Elmegreen, Elmegreen, \& Dressler (1982). To display the effect of the global environment on the arm classes more clearly, we plot cumulative distributions of arm classes in the low- and high- $L_{x}$ clusters as a function of $r / R_{v i r}$ in Fig. 10 where the upper panel shows the cumulative distributions of grand design plus multiple arms $(\mathrm{G}+\mathrm{M})$ while the lower panel displays those of the flocculent arms $(\mathrm{F})$. Clearly, there are considerable differences in the cumulative distributions of arm classes between the low- $L_{x}$ clusters and the high- $L_{x}$ clusters. The probability of K-S test for $\mathrm{G}+\mathrm{M}$ in low- $L_{x}$ and high- $L_{x}$ clusters (upper panel) is 0.04 and that for the $\mathrm{F}$ (lower panel) is 0.05, respectively, which indicates that both $\mathrm{G}+\mathrm{M}$ and $\mathrm{F}$ show significantly different behavior in the low- $L_{x}$ and high- $L_{x}$ clusters.

\subsection{Possible Mechanisms for the Morphology- Radius Relation}

There are a number of mechanisms that could be responsible for the observed morphology-radius relation for Hubble types. Some mechanisms such as ram pres- 

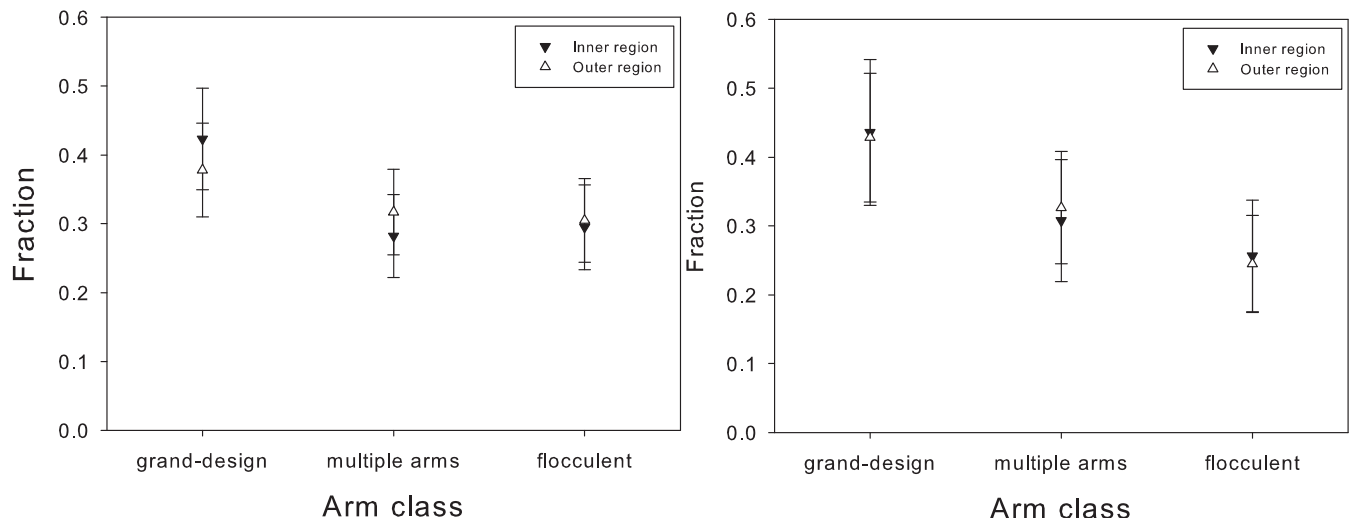

Fig. 7.- The number distribution of the arm classes of the low- $L_{x}$ clusters (left panel) and high- $L_{x}$ clusters (right panel). The galaxies in the inner regions $\left(0<r / R_{\text {vir }}<0.5\right)$ are plotted as solid triangles and those in the outer regions $\left.\left(0.5<r / R_{v i r}<1.0\right)\right)$ are plotted as open triangles. The error bar indicates the Poisson errors.
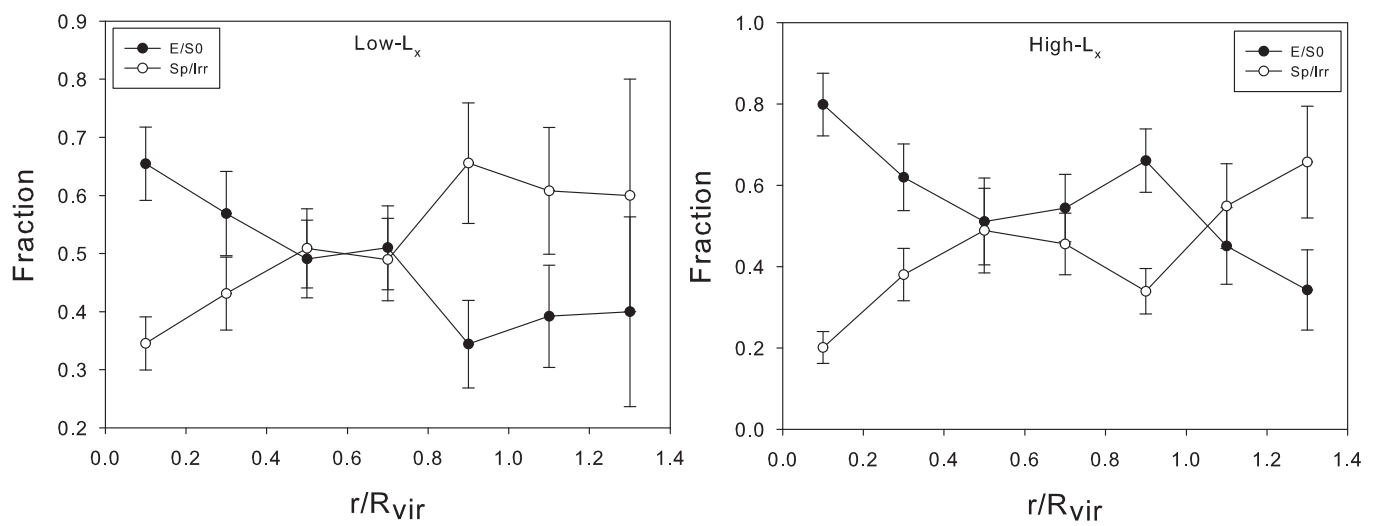

Fig. 8.- The distribution of Hubble types as a function of $r / R_{v i r}$. The left panel is for the low- $L_{x}$ clusters and the right panel is for the high- $L_{x}$ clusters.
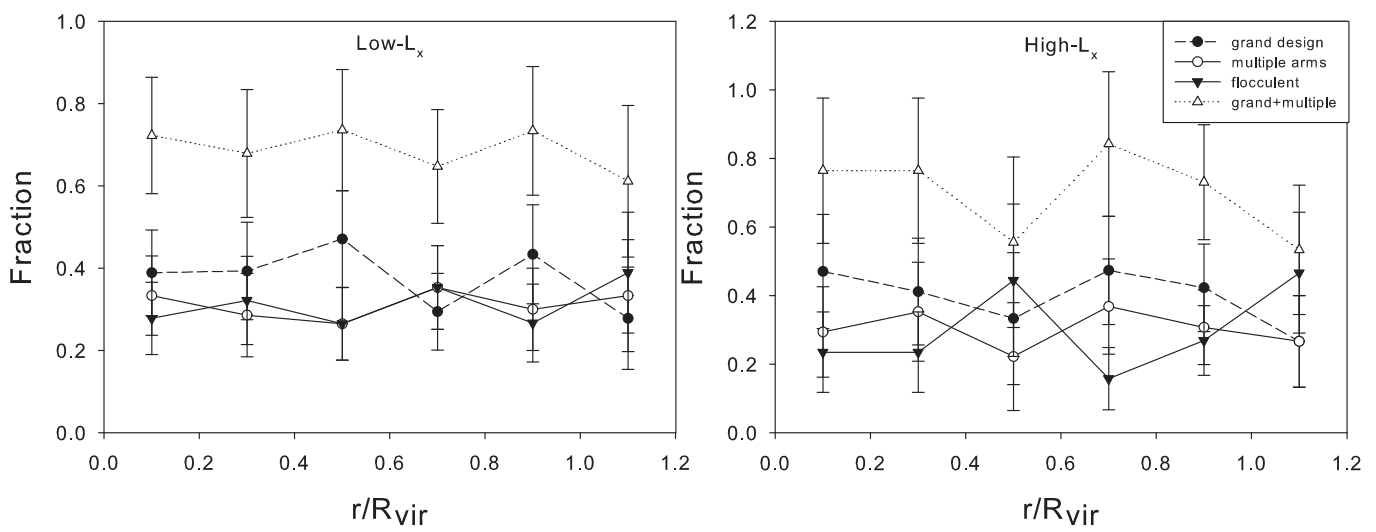

Fig. 9. - The distribution of arm classes as a function of $r / R_{v i r}$. The left panel is for the low- $L_{x}$ clusters and the right panel is for the high- $L_{x}$ clusters. The dotted line shows the fraction of the number of grand design and multiple arms added together. 


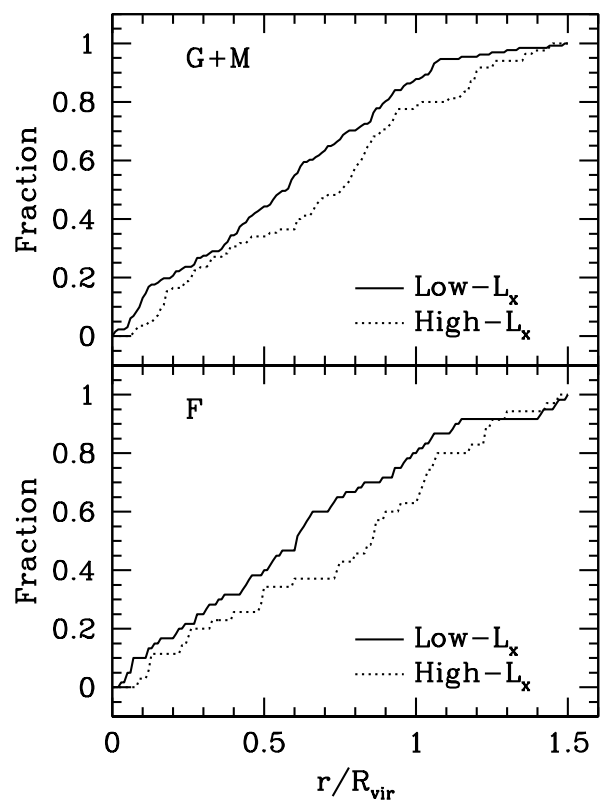

Fig. 10. - The cumulative distribution of arm classes as a function of $r / R_{\text {vir }}$. The upper panel displays the cumulative distributions of $\mathrm{G}+\mathrm{M}$ and the lower panel presents those of $\mathrm{F}$. The solid lines and dotted lines represent the low- $L_{x}$ clusters and the high- $L_{x}$ clusters, respectively.

sure stripping (Gunn \& Gott 1972) and thermal evaporation (Cowie \& Songaila 1977), and viscous stripping (Nulsen 1982) are criticized because of their inability to change the structures of galaxies even though they are efficient at shutting down the star formation activity (Park \& Hwang 2009). However, some of these are still viable for the explanation of the distribution of the arm classes in the cluster environment.

There are two features in the arm class distribution that deserve explanation. The first is the preponderance of grand design spirals especially in regions where tidal interactions are supposed to be large. The second is the increase in the fraction of flocculent spirals along the clustercentric radius. While the former was affected by the local and global environments, the latter exhibits a kind of morphology-radius relation for arm classes although it is significantly weaker than that of the Hubble-types.

In numerical simulations, multiple arms are transient features (Sellwood 2000; Bottema 2003). The number of arms $(m)$ is known to be a strong function of disk-to-halo mass (Sellwood \& Carlberg 1984). Carlberg \& Freedman (1985) showed that $m=1 / f$ where $f$ is the fractional contribution of the disk to the rotation curve. Thus, the grand design galaxies which are defined as galaxies with two symmetric arms $(m=2)$ have more massive disks than multiple arms $(m>2)$ and flocculent arms.

The fraction of grand design galaxies is higher than those of multiple armed and flocculent galaxies, which reaches about $40 \%$ in the both X-ray luminosity groups. Thus, in terms of disk mass, less massive disks dominate our sample. The reason is possibly that there are a variety of mechanisms to remove the gas from the disk of spiral galaxies in the environment of a cluster.

There are two pathways leading to the formation of a galaxy disk with low f. The first involves removal of some of the gas before it could settle in the plane of the disk to form stars. The second pathway involves tidal galaxy-galaxy interactions. Most hydrodynamic processes such as ram pressure stripping (Gunn \& Gott 1972) and thermal evaporation (Cowie \& Songaila 1977) are viable mechanisms for gas removal. Tidal interactions between galaxies are also known to remove gas in galaxies and transform late types to early types (Spitzer \& Baade 1951; Merritt 1983; Park \& Hwang 2009). Although this mechanism seems insufficient to remove gas completely due to the short duration of the interaction (Merritt 1984; Byrd \& Valtonen 1990), it does play a role in removing gas from the disk of a galaxy. Harassment (Moore et al. 1996; Moore, Lake, \& Katz 1998; Moore, Lake, Quinn, \& Stadel 1999) and galaxy-cluster interactions also play some role in the removal of gas before being converted to the stars.

During the classification procedure, we found that a considerable number of disk galaxies have very small disks. We are planning to study the structural parameters of these galaxies in detail in the future. This hypothesis can also be checked by computer simulations too.

Note that galaxies are affected by tidal forces due to the entire cluster (Merritt 1984; Byrd \& Valtonen 1990; Gnedin 2003). This tidal force will be stronger towards the cluster center, and will depend on the total mass, concentration of the mass and velocity dispersion, which are all in turn correlated with the X-ray luminosity of the cluster (Quintana \& Melnick 1982).

If global density waves are the main drivers of star formation in the cluster spirals, we expect a larger fraction of multiple armed galaxies than grand design arms. However, observations indicate the exact opposite: there seem to be mechanisms that suppress the formation of multiple arms. Considering that the majority of multiple armed galaxies are $\mathrm{AC} 6$ or $\mathrm{AC}$ 7 which are characterized by the ring-like structures rather than literally multiple armed spirals (AC 9), and that there are a number of lenticular galaxies associated with tidal debris, we suspect that some of the multiple armed galaxies are destroyed after formation by tidal interactions. This picture is consistent with Buta \& Combes (1996) who argued that rings are easily harassed by tidal forces and are unlikely to be in clusters. Thus tidal interactions reduce multiple armed spirals.

Given the above two observations, we can obtain an explanation for the nearly flat distribution of the frac- 
tion of grand design and multiple arm spirals together in Fig. 9. While tidal forces in clusters hinder the production of thick disks, which is needed for the formation of grand design arms, it also hinders the production of ring galaxies and removes multiple armed galaxies. We suspect that these two opposing mechanisms make a balance and produce the observed distribution of arm classes.

The deficiency of flocculent spirals in the present sample which is the most pronounced difference compared with field galaxies seems to be due to the combined effect of local environment and global environment. It is also worth noting that deficiency of late type spirals (Fig. 4) is closely related to the low fraction of flocculent spirals. Since late type spirals generally have larger amount of gas than the early type galaxies, the gas removal mechanisms that operate in the cluster environment play a critical role in leading to the deficiency of late type spirals.

\section{CONCLUSIONS}

We have studied the properties of galaxies in the 20 Abell clusters that were both in the SDSS survey region and the NORAS catalog, focusing on the dependence of the spiral morphology on the local and global environment. We used the clustercentric radius and X-ray luminosity as proxies of the local and global environment, respectively. Using $\mathrm{X}$-ray selected clusters, we defined a low- $L_{x}$ group $\left(6\right.$ clusters, $\left.L_{x}<0.15 \times 10^{44} \mathrm{erg} / \mathrm{s}\right)$ and high- $L_{x}$ group ( 3 clusters, $L_{x}>1.8 \times 10^{44} \mathrm{erg} / \mathrm{s}$ ) to see the effect of the global environment on the spiral morphology. Our major results are as follows.

1. The morphology-clustercentric radius relation is observed in 20 X-ray selected clusters, with some deviation near the cluster virial radius in high- $L_{x}$ clusters. The reason for this deviation is not yet understood but it suggests some role of the global environment on the morphology of galaxies, especially in the X-lay luminous clusters. Besides this, spiral galaxies are dominated by early type ones (earlier than Sc) in cluster environment. This trend is more apparent in the low$L_{x}$ cluster, especially in the inner regions.

2 . The grand design arms comprise about $40 \%$ of the cluster spiral galaxies, regardless of the cluster X-ray luminosity. Since dominant fractions of spiral galaxies are likely to have less massive disks in cluster environment, there seem to be some mechanism that suppresses the high- $m$ mode of star formation. We conjecture that strong tidal forces due to galaxy-galaxy interactions favor the low- $m$ mode of star formation that makes grand design spirals.

3. Flocculent spirals are highly deficient in cluster spirals and the local and global environment seem to work together in the suppression of flocculent spirals. The deficiency of flocculent and late type spirals in the cluster environment seems to be closely related to the gas removal mechanisms operated in galaxy clusters.
4. There is a dichotomy in the virialization of the clusters. While low- $L_{x}$ clusters are thought to be fully virialized, the high- $L_{x}$ clusters are not yet virialized. There seems to be a continuous infall of galaxies in the high- $L_{x}$ clusters.

\section{ACKNOWLEDGMENTS}

This work was partly supported by the NRF Research grant 2010-0023319.

\section{REFERENCES}

Abazajian, K., et al. 2009, The Seventh Data Release of the Sloan Digital Sky Survey, ApJS, 182, 543

Abell, G. O., Corwin, H. G., \& Olowin R. P. 1989, A Catalog of Rich Clusters of Galaxies, ApJ, 367, 64

Ann, H. B., Park, C., \& Choi, Y.-Y. 2008, Galactic Satellite Systems: Radial Distribution and Environment Dependence of Galaxy Morphology, MNRAS, 389,86

Balogh, M. L., Smail, I., Bower, R. G., Ziegler, B. L., Smith, G. P., Davies, R. L., Gaztelu, A., Kneib, J.-P., \& Ebeling, H. 2002, Distinguishing Local and Global Influences on Galaxy Morphology: A Hubble Space Telescope: Comparison of High and Low XRay Luminosity Clusters, ApJ, 566, 123

Blanton, M. R., Lin, H. L., Robert H., Maley, F. M., Young, N., Zehavi, I. , \& Loveday, J. 2003, An Efficient Targeting Strategy for Multiobject Spectrograph Surveys: the Sloan Digital Sky Survey "Tiling" Algorithm, AJ, 125, 2276

Bohringer, H., Voges, W., Huchra, J. P., McLean, B., Giacconi, R., Rosati, P., Burg, R., Mader, J., Schuecker, P., \& Simic, D. 2000, The Northern ROSAT All-Sky (NORAS) Galaxy Cluster Survey. I. X-Ray Properties of Clusters Detected as Extended X-Ray Sources, ApJS, 129, 43

Buta, R., \& Combes, F. 1996, Galactic Rings, Fundamentals of Cosmic Physics, 17, 95

Bottema, R. 2003, Simulations of Normal Spiral Galaxies, MNRAS, 344, 358

Byrd, G., \& Valtonen, M. 1990, Tidal Generation of Active Spirals and S0 Galaxies by Rich Clusters, ApJ, 350, 89

Cartin, D., \& Khanna, G. 2002, Self-Regulated Model of Galactic Spiral Structure Formation, Physical Rev. E. 65, 6160

Carlberg, R. G., \& Freedman, W. L. 1985, Dissipative Models of Spiral Galaxies, ApJ, 298, 486

Carlberg, R. G., Yee, H. K. C., \& Ellingson, E. 1997, The Average Mass and Light Profiles of Galaxy Clusters, ApJ, 478, 462 
Castander, F. J., et al. 2001, The First Hour of Extragalactic Data of the Sloan Digital Sky Survey Spectroscopic Commissioning: The Coma Cluster, AJ, 121,2331

Choi, Y.-Y., Han, D.-H., Kim, S. S. 2010, Korea Institute for Advanced Study Value-Added Galaxy Catalog, JKAS, 43, 191

Cowie, L. L., \& Songaila, A. 1977, Thermal Evaporation of Gas within Galaxies by a Hot Intergalactic Medium, Nature, 266, 501

de Vaucouleurs, G. 1959, Classification and Morphology of External Galaxies, HDP, 53, 275

de Vaucouleurs, G. 1961, Recent Studies of Clusters and Superclusters, ApJS, 5, 233

Dobbs, C. L., Theis, C., Pringle, J. E., \& Bate, M. R. 2010, Simulations of the Grand Design Galaxy M51: a Case Study for Analyzing Tidally Induced Spiral Structure, MNRAS, 403, 625

Dressler, A. 1980, Galaxy Morphology in Rich Clusters - Implications for the Formation and Evolution of Galaxies, ApJ, 236, 351

Elmegreen, D. M., \& Elmegreen, B. G. 1982, Flocculent and Grand Design Spiral Structure in Field, Binary and Group Galaxies, MNRAS, 201, 1021

Elmegreen, D. M. Elmegreen, B. G., \& Dressler, A. 1982, Flocculent and Grand Design Spiral Arm Structure in Cluster Galaxies, MNRAS, 201, 1035

Elmegreen, D. M. \& Elmegreen, B. G. 1987, Arm Classifications for Spiral Galaxies, ApJ, 314, 3

Elmegreen, B. G. 1990, Grand Design, Multiple Arm, and Flocculent Spiral Galaxies, NYASA, 596, 40

Elmegreen, B. G., Elmegreen, D. M., \& Leitner S. N. 2003, A Turbulent Origin for Flocculent Spiral Structure in Galaxies, ApJ, 590, 271

Fadda, D., Girardi, M., Giuricin, G., Mardirossian, F., \& Mezzetti, M. 1996, The Observational Distribution of Internal Velocity Dispersions in Nearby Galaxy Clusters, ApJ, 473, 670

Fukugita, M., Ichikawa, T., Gunn, J. E., Doi, M., Shimasaku, K., \& Schneider, D. P. 1996, The Sloan Digital Sky Survey Photometric System, AJ, 111, 1748

Gerola, H., \& Seiden, P. E. 1978, Stochastic Star Formation and Spiral Structure of Galaxies, ApJ, 223, 129

Goto, T., Yamauchi, C., Fujita, Y., Okamura, S., Sekiguchi, M., Smail, I., Bernardi, M., \& Gomez, P. L. 2003, The Morphology-Density Relation in the Sloan Digital Sky Survey, MNRAS, 346, 601

Gray, M. E., Wolf, C., Meisenheimer, K., Taylor, A., Dye, S., Borch, A., \& Kleinheinrich, M. 2004, Linking Star Formation and Environment in the A901/902 Supercluster, MNRAS, 347, L73
Gnedin, O. Y. 2003, Tidal Effects in Clusters of Galaxies, ApJ, 582, 141

Gunn, J. E., \& Gott, J., Richard, III. 1972, On the Infall of Matter Into Clusters of Galaxies and Some Effects on Their Evolution, ApJ, 176, 1

Gunn, J. E., et al. 1998, The Sloan Digital Sky Survey Photometric Camera, AJ, 116, 3040

Helsdon, S. F., \& Ponman, T. J. 2003, The Morphology-Density Relation in X-Ray-Bright Galaxy Groups, MNRAS, 339, 29

Hogg, D. W., Finkbeiner, D. P., Schlegel, D. J., \& Gunn, J. E. 2001, A Visual Photometricity and Extinction Monitor at the Apache Point Observatory, AJ, 122,2129

Hubble, E. 1936, Realm of the Nebulae, Hubble. New Haven: Yale University Press

Ivezic, Z., et al. 2004, SDSS Data Management and Photometric Quality Assessment, Astronomische Nachrichten, 325, 583

Lin, C. C., \& Shu, Frank H. 1964, On the Spiral Structure of Disk Galaxies, ApJ, 140, 646

Merritt, D. 1983, Relaxation and Tidal Stripping in Rich Clusters of Galaxies. I. Evolution of the Mass Distribution, ApJ, 264, 24

Merritt, D. 1984, Relaxation and Tidal Stripping in Rich Clusters of Galaxies. II. Evolution of the Luminosity Distribution, ApJ, 276, 26

Moore, B., Katz, N., Lake, G., Dressler, A., \& Oemler, A. 1996, Galaxy Harassment and the Evolution of Clusters of Galaxies, Nature, 379, 613

Moore, B., Lake, G., \& Katz, N. 1998, Morphological Transformation from Galaxy Harassment, ApJ, 495, 139

Moore, B., Lake, G., Quinn, T., \& Stadel, J. 1999, On the Survival and Destruction of Spiral Galaxies in Clusters, MNRAS, 304, 465

Mueller, M. W., \& Arnett, W. D. 1976, Propagating Star Formation and Irregular Structure in Spiral Galaxies, ApJ, 210, 670

Muldrew, S. et al. 2011, Measures of Galaxy Environment I - What is "Environment"?, arXiv1109.6328M

Nulsen, P. E. J. 1982, Transport Processes and the Stripping of Cluster Galaxies, MNRAS, 198, 1007

Oh, S. H., Kim, W.-T., Lee, H. M., \& Kim, J. 2008, Physical Properties of Tidal Features in Interacting Disk Galaxies, ApJ, 683, 94

Park, C., \& Hwang, H. S. 2009, Interactions of Galaxies in the Galaxy Cluster Environment, ApJ, 699, 1599

Park, C., \& Choi, Y.-Y. 2005, Morphology Segregation of Galaxies in Color-Color Gradient Space, ApJ, 635, L29 
Park, C., Choi, Y.-Y., Vogeley, M. S., Gott, J. R. III., \& Blanton, M. R. 2007, Environmental Dependence of Properties of Galaxies in the Sloan Digital Sky Survey, ApJ, 658, 898

Peebles, P. J. E. 1993, Principles of Physical Cosmology, Princeton University Press, Princeton

Pier, J. R., Munn, J. A., Hindsley, R. B., Hennessy, G. S., Kent, S. M., Lupton, R. H., \& Ivezic, Z. 2003, Astrometric Calibration of the Sloan Digital Sky Survey, AJ, 125, 1559

Postman, M., et al. 2005, The Morphology-Density Relation in $\mathrm{z} \sim 1$ Clusters, ApJ, 623, 712

Postman, M., \& Geller, M. J. 1984, The MorphologyDensity Relation - The Group Connection, ApJ, 281, 95

Quintana, H., \& Melnick, J. 1982, The Correlation between X-Ray Luminosity and Velocity Dispersion in Clusters of Galaxies, AJ, 87, 972

Sandage, A. 1961, The Hubble Atlas of Galaxies, Washington: Carnegie Institution

Seiden, P. E., \& Gerola, H. 1982, Propagating Star Formation and the Structure and Evolution of Galaxies, Fund. Cosmic Phys., 7, 241

Sellwood, J. A. 2000, Spiral Structure as a Recurrent Instability, AP\&SS, 272, 31

Sellwood, J. A., \& Carlberg, R. G. 1984, Spiral Instabilities Provoked by Accretion and Star Formation, ApJ, 282, 61

Spitzer, L. Jr., \& Baade, W. 1951, Stellar Populations and Collisions of Galaxies, ApJ, 113, 413

Stoughton, C., et al. 2002, Sloan Digital Sky Survey: Early Data Release, AJ, 123, 485

Thomas, T., \& Katgert, P. 2006, The ESO nearby Abell Cluster Survey. IX. The Morphology-Radius and Morphology-Density Relations in Rich Galaxy Clusters, A\&A, 446, 31

Toomre, A., \& Toomre, J. 1972, Galactic Bridges and Tails, ApJ, 178, 623

Tran, K.-V. H., Simard, Luc Z., Ann I. M., John S. 2001, The Galaxy Populations of X-Ray-detected, Poor Groups, ApJ, 549, 172

Tucker, D. L., et al. 2006, The Sloan Digital Sky Survey Monitor Telescope Pipeline, Astronomische Nachrichten, 327, 821

Tully, R. B. 1974, The Kinematics and Dynamics of M51. III. The Spiral Structure, ApJS, 27, 251

Uomoto, A., et al. 1999, Bulletin of the American Astronomical Society, 31, 1501

van den Bergh, S. 2002, Spiral Structure and Galaxy Environment, AJ, 124, 786

Whitmore, B. C. 1995, What Determines the Morphological Fractions in Groups and Clusters?, ASPC, 70,41
Whitmore, B. C., \& Gilmore, D. M, 1991, On the Interpretation of the Morphology-Density Relation for Galaxies in Clusters, ApJ, 367, 64

Wolf, C., et al. 2009, The STAGES View of Red Spirals and Dusty Red Galaxies: Mass-Dependent Quenching of Star Formation in Cluster Infall, MNRAS, 393,1302

York, D. G., et al. 2000, The Sloan Digital Sky Survey: Technical Summary, AJ, 120, 1579

\section{APPENDIX A. Sample images of spiral arm morphologies}

We display color images which show the arm morphology in Fig. 11-12. Fig. 11 shows the sample images of galaxies brighter than $r=15$ while Fig. 12 displays those for galaxies fainter than $r=16.5$ 


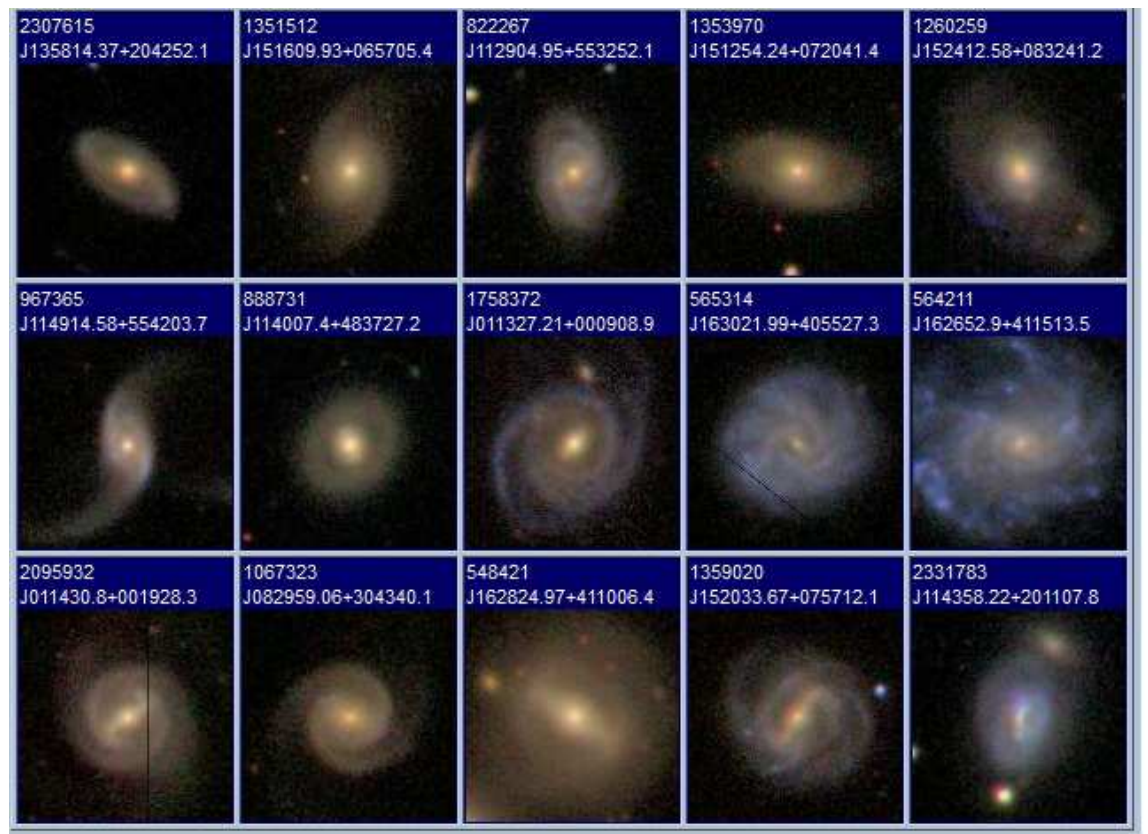

Fig. 11. - Sample images of 15 galaxies which are brighter than $r=15$. The top line, middle line and bottom line show the sample images of SA, SAB, and SB galaxies, respectively. The spiral morphology of these galaxies, as given in Table 3, are as follows. From left to right, $(1,1,1),(1,2,1),(1,1,2),(1,1,2),(1,2,3)$. $(1,2,3)$ for the top line, $(2,2,1),(2,1$, $2),(2,1,2),(2,2,2),(2,2,3)$ for the middle line, and $(3,1,1),(3,2,1),(3,1,2),(3,1,2),(3,1,3)$ for the bottom line.

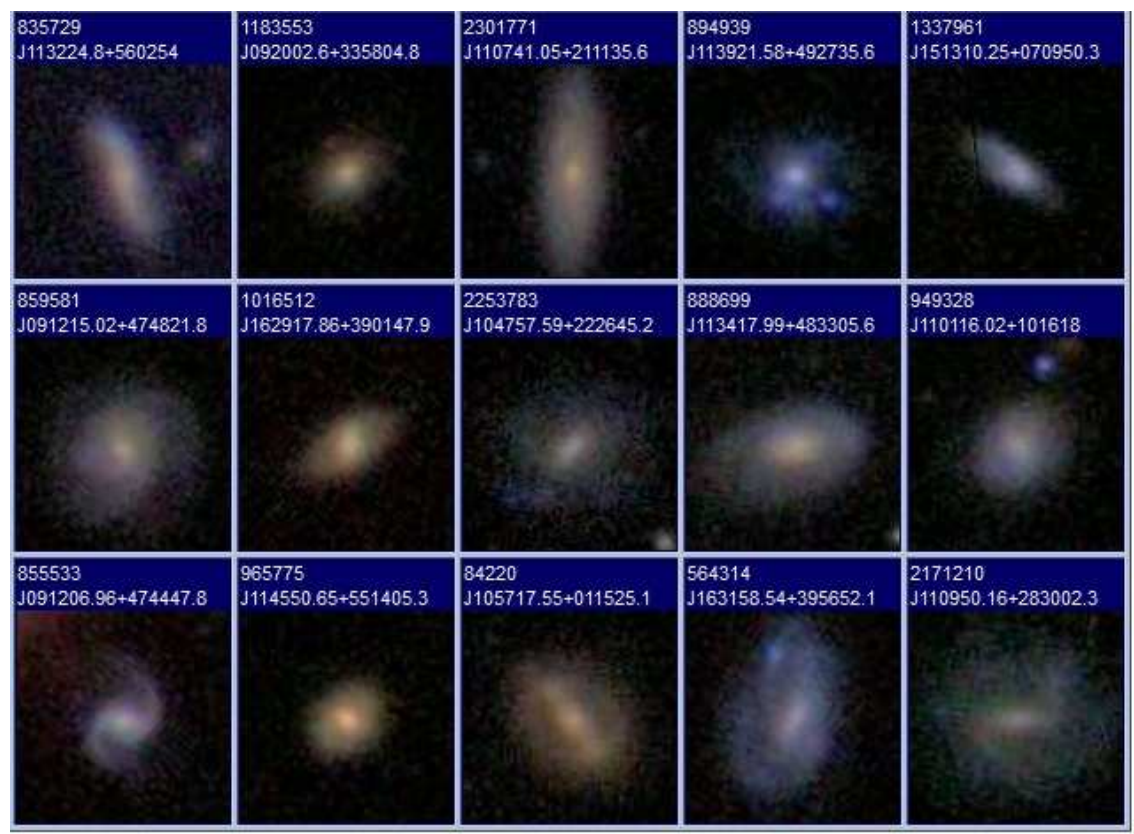

Fig. 12. - The same as in Fig. 11 but for galaxies fainter than $r=16.5$ with two times smaller pixel scales to zoom in. From left to right, $(1,2,1),(1,1,2),(1,2,2),(1,1,3),(1,2,3)$ for the top line, $(2,2,1),(2,1,2),(2,2,2),(2,1,3),(2$, $3,3)$ for the middle line, and $(3,2,1),(3,1,1),(3,1,2),(3,2,3),(3,2,3)$ for the bottom line. 